\title{
Leaders
}

\section{Overuse injuries: where to now?}

An overuse injury occurs when repetitive microtrauma overloads the capacity of a tissue to repair itself. This may result in an inflammatory response leading to acute, and then possibly chronic, inflammation, ultimately resulting in structural changes in tissue. Overuse injuries have become an increasing problem in sports medicine in the past two decades as a result of a trend towards increased volume of training in all sports.

In most primary care or referral-based sports medicine practices, overuse injuries comprise most presentations to the sports physician. In our recent 12 month survey of patients presenting to a sports medicine clinic in Melbourne, Australia, overuse injuries outnumbered acute injuries by a ratio of $2: 1 .^{1}$ The high incidence of overuse injuries was highlighted in our prospective study looking at a group of track and field runners over 12 months. In this study of 95 track and field athletes, $72 \%$ of all injuries were overuse injuries. ${ }^{2}$ Both the men and women in this group had an annual incidence of stress fracture of $20 \%$.

Our understanding of the pathology of the processes involved in most overuse injuries has increased dramatically in the past few years. If we look at bony injuries, we now understand that there is a continuum of bone stress ranging from normal bone to an actual stress fracture. It is the role of the clinician to determine clinically and with the aid of imaging where in that continuum of bone stress each patient's injury lies and adjust the treatment accordingly. Similarly, with tendon injuries we are now aware that the previous description of these injuries as "tendinitis" was not entirely accurate and that in many of these longstanding cases the pathological process is more degenerative than inflammatory. This has caused us to adjust our treatment away from reducing inflammation towards direct treatment either by physiotherapy or surgery to decrease load and stimulate the healing response.

We have made considerable progress in the treatment and rehabilitation of athletes with overuse injuries. Prevention is, however, better than cure. Before we can be successful in the prevention of injuries we need to understand the causative factors and try to modify them. Establishing risk factors for the development of overuse injuries is our next challenge. Predisposing factors to the development of overuse injuries are often cited and include training errors, change of surface, inappropriate footwear, hormonal, nutritional and psychological fac- tors, malalignment, lack of flexibility, muscle imbalances, and weaknesses. ${ }^{4}$ However, there has been little research to confirm these risk factors and even less research involving intervention to modify or eliminate risk factors.

Our prospective study looking at possible risk factors in the development of stress fractures in track and field athletes identified low bone density, late menarche, and reduced calf girth as significant risk factors for the development of stress factures in women. ${ }^{5}$ None of the possible risk factors in the men in this study reached statistical significance. Further work must be done assessing risk factors in other overuse injuries, particularly tendon injuries. Once possible risk factors are identified, intervention studies can be designed.

The clinician must establish a cause(s) for every overuse injury. It is not sufficient to diagnose and treat the injury then to return the athlete to his or her previous activity without modification. Risk factors need to be modified (for example, reduction of training, increased cross training, use of orthotics, hormonal supplementation) to ensure that the injury does not recur. It is essential to include the athlete's coach or trainer in the rehabilitation programme and to ensure that they understand the factors that have led to this injury. Without their support all the medical advice to the athlete is useless.

Most overuse injuries should be preventable, but we need to understand better the factors causing them and how we can modify these factors.

Olympic Park Sports Medicine Centre

PETER BRUKNER

Melbourne, Australia

KIM BENNELI

School of Physiotherapy

Faculty of Medicine, Dentistry and Health Sciences

University of Melbourne

Parkville, Australia

1 Baquie P, Brukner PD. Injuries presenting to an Australian sports medicine clinic. Clin 7 Sport Med (in press)

2 Bennell KL, Crossley K. Musculoskeletal injuries in track and field: incidence, distribution and risk factors. Aust f Sci Med Sport 1996;28:69-75. dence, distribution and risk factors. Aust $\mathcal{F}$ Sci Med Sport 1996;28:69-75.
Bennell KL, Malcolm SA, Thomas SA, et al. The incidence and distribution of stress fracture in competition track and field athletes. A twelve month study. Am $\mathcal{F}$ Sports Med 1996;24:211-18.

4 Brukner P, Khan K. Clinical sports medicine. Sydney: McGraw Hill, 1993 17.

5 Bennell KL, Malcolm SA, Thomas SA, et al. Risk factors for stress factors in track and field athletes. A twelve month prospective study. $A m \mathcal{F}$ Sport Med (in press). 